\title{
POLYMORPHISM IN THE AUSTRALIAN ACRIDIDAE \\ II. CHANGES IN COLOUR PATTERN GENE FREQUENCIES IN THE PLAGUE LOCUST, CHORTOICETES TERMINIFERA
}

\author{
O. R. BYRNE
}

Botany Department, A.N.U., Canberro

Received 14.ii.67

\section{INTRODUCTION}

Mathematical theory in population genetics formulated by Fisher, Haldane and Wright during the I93o's paved the way for intensive studies of evolutionary processes in both laboratory and natural populations of plants and animals. It is particularly true in many polymorphic situations that selective pressures of a much higher order than at first postulated are operative, bringing about remarkable genetic changes in a relatively short time, as in industrial melanism (Kettlewell, I956).

In most species where polymorphism has been investigated (for example colour patterns in adult butterflies, beetles or gene arrangements in larvæ of Drosophila) it is possible to estimate gene frequencies only for a particular stage in the life-cycle. For such organisms information on the net effect of selection operating throughout their life-cycle can be obtained. It is often desirable to know if a particular selective value is relatively constant throughout the life-cycle or if it is operative only during a certain stage of development, e.g. adult or pupal stage. In grasshoppers, colour patterns which appear during the first nymphal instar and remain obvious through the life of the insect provide an excellent opportunity to study the immediate effect of natural selection.

No detailed study of the population genetics of colour patterns in the Orthoptera has been made although inheritance studies in some species have been carried out. Fisher's (I930, I939) analysis of Nabour's extensive breeding data indicated that in Apotettix eurycephalus, heterozygotes had a selective advantage of 7 per cent. over the dominant homozygotes and about 5 per cent. over the common recessive form. Also from the data for natural populations of Paratettix texanus Fisher showed that the frequency of individuals heterozygous for two or more dominant genes was less than expected and he suggested that selective forces of the order of 40 per cent. were operating against these forms in the field. Rubtzov (I935) reported relative frequencies of four different colour patterns in Chorthippus albomarginatus from habitats which varied from dry to humid. His data suggest that in moist habitats, green individuals have a selective advantage over brown forms whereas in dry habitats the situation is reversed. There were also marked differences in size and behaviour between the two forms.

The Australian plague locust, Chortoicetes terminifera, is polymorphic 
for at least nine colour patterns (Byrne, I967). These colour patterns are inherited as though determined by four multiple alleles, $F^{a}, F^{n}, F^{r}$ and $F^{t}$. Heterozygotes can be recognised except in the case of $F^{a} F^{r}$, which is indistinguishable from $F^{a} F^{a}$.

\section{MATERIAL AND METHODS}

Six separate populations located in the central western district of New South Wales were sampled between October 1960 and March 196r. Each population was sampled weekly during the nymphal stage and fortnightly during the adult stage of the first generation. Only two of these (Pop. A and Pop. B) produced a second generation with adequate numbers of individuals for study. Difficulties in publication of extensive tables of data do not permit a detailed treatment of all the data

TABLE I

Test for heterogeneity of colour patterns among replicate samples using three sampling methods

\begin{tabular}{|l|c|c|c|c|}
\hline \multicolumn{1}{|c|}{ Method } & No. of reps. & D.F. & Chi-square & $\mathrm{P}$ \\
\hline Suction & 4 & 13 & $2 \mathrm{I} \cdot 4$ & $0.1 \mathrm{P} 0.05$ \\
Net & 2 & 6 & 5.3 & $0.5 \mathrm{P} 0.3$ \\
Cage & 2 & 6 & 3.1 & $0.7 \mathrm{P} 0.8$ \\
\hline
\end{tabular}

collected from the six populations. This paper will consider only changes in colour pattern frequency in populations $\mathrm{A}$ and $\mathrm{B}$ for which more extensive data are available. However, except for some minor irregularities, the same general trend in changes in colour pattern frequency occurred in all populations.

Samples were obtained using three methods. First instar nymphs are relatively inactive for the first few days and they rarely hop high enough from the ground to be caught by sweeping with a hand net. It was found that adequate samples of these individuals could be obtained by sucking them into a flask. After most individuals in a population had completed their first instar, a hand net was used for sampling. However, it was difficult to collect adequate numbers of adults in this way unless they were in a fairly dense population. The third sampling method involved using a cage attached to the front of a car. This was used to capture adults wherever possible.

Naturally, only one sampling method was used on any one date. Replicate samples obtained by each method were homogeneous with respect to colour pattern frequency. This indicates that on each occasion random samples of the populations were obtained. Values of chi-square for heterogeneity are given in table $\mathrm{I}$.

\section{LIFE HISTORY UNDER NATURAL CONDITIONS}

A generation of Chortoicetes may be completed within 7 weeks under laboratory conditions (Byrne, I967). Swan (1956) has recorded a generation time of I I weeks under favourable conditions in the field. During the studies reported here, considerable data on generation time and developmental rates for nymphal instars were obtained. In population A, development in which was typical of the populations studied, 
hatching commenced on 30 th September, after a fall of rain on 23rd September. The relative frequencies of the six different instars for males and females collected from population $\mathrm{A}$ at the different dates are given in table 2. First instar nymphs were captured up to 26 days after the commencement of hatching. Therefore, hatching must have continued for about three weeks even though no further rainfall was

TABLE 2

Relative frequencies of the six instars among males and females from population $A$

\begin{tabular}{|c|c|c|c|c|c|c|c|c|}
\hline \multirow{2}{*}{\multicolumn{2}{|c|}{ Instar }} & \multicolumn{7}{|c|}{ No. of days after start of hatching } \\
\hline & & 4 & I I & I 9 & 26 & 33 & 47 & 60 \\
\hline & o & $\begin{array}{l}0.91 \\
0.95\end{array}$ & $\begin{array}{l}0.87 \\
0.87\end{array}$ & $\begin{array}{l}0 \cdot 34 \\
0 \cdot 3^{2}\end{array}$ & $\begin{array}{l}0.05 \\
0.03\end{array}$ & $\begin{array}{l}0.01 \\
0.00\end{array}$ & $\cdots$ & $\begin{array}{l}\cdots \\
\cdots\end{array}$ \\
\hline 2 & $\begin{array}{l}\text { q } \\
\text { ot }\end{array}$ & $\begin{array}{l}0.09 \\
0.05\end{array}$ & $\begin{array}{l}0.12 \\
0.12\end{array}$ & $\begin{array}{l}0.33 \\
0.40\end{array}$ & $\begin{array}{l}0.42 \\
0.17\end{array}$ & $\begin{array}{l}0.04 \\
0.01\end{array}$ & $\begin{array}{l}\cdots \\
\cdots\end{array}$ & $\begin{array}{l}\cdots \\
\cdots\end{array}$ \\
\hline 3 & $\begin{array}{l}\text { q } \\
\text { d }\end{array}$ & $\begin{array}{l}\cdots \\
\cdots\end{array}$ & $\begin{array}{l}0.01 \\
0.01\end{array}$ & $\begin{array}{l}0.28 \\
0.17\end{array}$ & $\begin{array}{l}0.28 \\
0.19\end{array}$ & $\begin{array}{l}0 \cdot 20 \\
0.02\end{array}$ & $\begin{array}{l}\cdots \\
\cdots\end{array}$ & $\cdots$ \\
\hline 4 & $\begin{array}{l}\text { q } \\
\text { a }\end{array}$ & $\begin{array}{l}\cdots \\
\cdots\end{array}$ & $\cdots$ & $\begin{array}{l}0.05 \\
0.11\end{array}$ & $\begin{array}{l}0.23 \\
0.59\end{array}$ & $\begin{array}{l}0.43 \\
0.3^{8}\end{array}$ & $\begin{array}{l}0 \cdot 01 \\
0 \cdot 00\end{array}$ & $\begin{array}{l}\cdots \\
\cdots\end{array}$ \\
\hline 5 & $\begin{array}{l}\text { o } \\
0\end{array}$ & $\begin{array}{l}\cdots \\
\cdots\end{array}$ & $\begin{array}{l}\cdots \\
\cdots\end{array}$ & $\begin{array}{l}\cdots \\
\cdots\end{array}$ & $\begin{array}{l}0.02 \\
0.02\end{array}$ & $\begin{array}{l}0.3 I \\
0.5^{8}\end{array}$ & $\begin{array}{l}0.15 \\
0.03\end{array}$ & $\cdots$ \\
\hline 6 & $\frac{q}{\partial}$ & $\begin{array}{l}\cdots \\
\cdots\end{array}$ & $\cdots$ & $\begin{array}{l}\cdots \\
\cdots\end{array}$ & $\begin{array}{l}\cdots \\
\cdots\end{array}$ & $\begin{array}{l}\text { O.OI } \\
\text { O.OI }\end{array}$ & $\begin{array}{l}0.84 \\
0.97\end{array}$ & $\begin{array}{l}I \cdot O \\
I \cdot O\end{array}$ \\
\hline $\begin{array}{l}\text { P'tio } \\
\text { S.E. }\end{array}$ & & $\begin{array}{l}0.50 \\
0.02\end{array}$ & $\begin{array}{l}0.49 \\
0.02\end{array}$ & $\begin{array}{l}0.476 \\
0.0231\end{array}$ & $\begin{array}{l}0.45 \text { I } \\
0.0279\end{array}$ & $\begin{array}{l}0.409 \\
0.0273\end{array}$ & $\begin{array}{l}0.329 \\
0.0233\end{array}$ & $\begin{array}{l}0.576 \\
0.0177\end{array}$ \\
\hline
\end{tabular}

recorded. More than half the males were adult by the forty-fourth day and after 8 weeks the population consisted almost entirely of adults. The second generation emerged on 18 th December, approximately i I weeks after the first generation.

For the first two weeks after hatching both sexes developed at approximately the same rate. However, after the third week most of the males were fourth and fifth instar and the females were second and third instar. This increase in rate of development in males was maintained and they were adult a week earlier on the average than females.

Active stages of $C$. terminifera rarely survive the winter period, which is normally passed in the egg stage. Eggs which are laid during autumn commence hatching in spring after the onset of warm weather and rainfall. In the areas most favourable for locusts, three generations may be completed in the one season. 


\section{DESCRIPTION OF THE HABITATS}

Populations $\mathrm{A}$ and $\mathrm{B}$ were located approximately 20 miles apart in central western New South Wales. In describing the habitats, particular reference is made to those temporal changes in the environment which appear to be most relevant to the changes in frequency of colour patterns.

Population $A$ (io miles south-south-west of Coonamble). The soil is sandy loam with rather small isolated areas of compact soil. A shallow, expansive watercourse runs along the southern end of the site.

When hatching commenced on ist October I96o, the vegetation was mostly Medicago denticulata and dormant tussocks of Stipa aristaglumis. The annual medic provided a green cover for the nymphs until mid-November, by which time it had died. The background colour of the habitat changed from green at hatching time to brown within six weeks. After December 196o, the perennial grass Stipa aristaglumis grew vigorously. Collecting was done over an area of about 12 acres.

Population $B$ (8 miles north-west of Coonamble). This site has welldefined oviposition and food-shelter habitats. The soil on the oviposition habitat is sandy and compact. Plant species included Eucalyptus populnea, Cynodon dactylon and Argemone mexicana.

The food-shelter habitat is on lower ground than the oviposition site. It has black, self-mulching soil. The only plants were Bassia quinquecuspis and a few plants of Astrebla lappacea.

When hatching started on ist October ig6o, the only food on the oviposition site was couch grass which covered a portion of the ground. Medic, which was abundant at the site for population A, was absent. Notwithstanding the arid appearance of this habitat, the population survived to the adult stage. However, even after summer rains, there was inadequate food on both oviposition and food-shelter habitats and the majority of adults migrated from the site.

\section{CHANGE IN COLOUR PATTERN FREQUENCY}

Table 3 shows the relative frequencies of colour pattern genotypes for populations $\mathrm{A}$ and $\mathrm{B}$ which were sampled at intervals during the first generation. The dates on which samples were collected are expressed in number of days after the date on which hatching was observed to commence. The size of the samples are given in the extreme right-hand column.

Out of $525^{2}$ locusts, $239^{2}$ were females. Sex was not recorded for first instars because external genitalia are not obvious at this stage. To maintain uniformity in presentation of the data, it was assumed that the sex ratio among first instars is $I: I$ and that colour patterns are distributed equally among first instar males and females. However, among each of the later instars it was found that males and females had different colour pattern frequencies and therefore the data for each sex are analysed separately. 
TABLE $3(a)$

Frequency of colour pattern genotypes in samples from population $A$

\begin{tabular}{|c|c|c|c|c|c|c|c|c|c|c|}
\hline \multirow{2}{*}{$\begin{array}{c}\text { Days after } \\
\text { hatching }\end{array}$} & \multicolumn{9}{|c|}{ Genotype } & \multirow{2}{*}{$\begin{array}{l}\text { No. in } \\
\text { sample }\end{array}$} \\
\hline & $\begin{array}{ll}a & a \\
a & r\end{array}$ & $a n$ & $a t$ & $n n$ & $n r$ & $n t$ & $r r$ & $r t$ & $t t$ & \\
\hline $4 \frac{9}{8}$ & \begin{tabular}{|l|}
0.021 \\
0.028
\end{tabular} & $\begin{array}{l}0.070 \\
0.06 \mathrm{I}\end{array}$ & $\begin{array}{l}0 \cdot 021 \\
0 \cdot 039\end{array}$ & $\mid \begin{array}{l}0 \cdot 129 \\
0 \cdot 1344\end{array}$ & $\begin{array}{l}0.215 \\
0.229\end{array}$ & $\begin{array}{l}0.215 \\
0.162\end{array}$ & $\begin{array}{l}\text { O. I } 400 \\
\text { O. I } 45\end{array}$ & $\begin{array}{l}0 \cdot 134 \\
0 \cdot 15\end{array}$ & $\begin{array}{l}0.054 \\
0 \cdot 050\end{array}$ & $\begin{array}{l}\text { I } 86 \\
\text { I } 79\end{array}$ \\
\hline II $\underset{0}{q}$ & $\begin{array}{l}0.020 \\
0.030\end{array}$ & $\begin{array}{l}0.085 \\
0.074\end{array}$ & $\begin{array}{l}0 \cdot 020 \\
0.025\end{array}$ & $\begin{array}{l}0.141 \\
0.129\end{array}$ & $\begin{array}{l}0.286 \\
0.267\end{array}$ & $\begin{array}{l}0 \cdot 15 \text { I } \\
0 \cdot 139\end{array}$ & $\begin{array}{l}0.095 \\
0.099\end{array}$ & $\begin{array}{l}\text { o. } 156 \\
0 \cdot 198\end{array}$ & $\begin{array}{l}0.035 \\
0.040\end{array}$ & $\begin{array}{l}199 \\
202\end{array}$ \\
\hline $19 \frac{9}{0}$ & $\begin{array}{l}0.018 \\
0.032\end{array}$ & $\begin{array}{l}0.037 \\
0.024\end{array}$ & $\begin{array}{l}0.023 \\
0 \cdot 040\end{array}$ & \begin{tabular}{|l|}
0.090 \\
0.089
\end{tabular} & $\begin{array}{l}0 \cdot 308 \\
0 \cdot 275\end{array}$ & $\begin{array}{l}0 \cdot 108 \\
0 \cdot 109\end{array}$ & $\begin{array}{l}0.077 \\
0.093\end{array}$ & $\begin{array}{l}0.285 \\
0.296\end{array}$ & $\begin{array}{l}0 \cdot 054 \\
0 \cdot 040\end{array}$ & $\begin{array}{l}221 \\
247\end{array}$ \\
\hline $26 \stackrel{9}{0}$ & $\begin{array}{l}0.007 \\
0.023\end{array}$ & $\begin{array}{l}0.022 \\
0.006\end{array}$ & $\begin{array}{l}0.037 \\
0.076\end{array}$ & $\begin{array}{l}0 \cdot 104 \\
0 \cdot 05^{8}\end{array}$ & $\begin{array}{l}0.244 \\
0.215\end{array}$ & $\begin{array}{l}0 \cdot 193 \\
0 \cdot 145\end{array}$ & $\begin{array}{l}0.133 \\
0.116\end{array}$ & $\begin{array}{l}0.230 \\
0.256\end{array}$ & $\begin{array}{l}0.030 \\
0 \cdot 104\end{array}$ & $\begin{array}{l}135 \\
\text { I } 72\end{array}$ \\
\hline 33 웅 & $\begin{array}{l}0.009 \\
0.025\end{array}$ & $\begin{array}{l}0.018 \\
0.006\end{array}$ & $\begin{array}{l}0.045 \\
0.019\end{array}$ & $\mid \begin{array}{l}0 \cdot 100 \\
0 \cdot 100\end{array}$ & $\begin{array}{l}0 \cdot 164 \\
0 \cdot 200\end{array}$ & $\begin{array}{l}0 \cdot 218 \\
0 \cdot 100\end{array}$ & \begin{tabular}{|l} 
O. I I 54 \\
O- I I9
\end{tabular} & $\begin{array}{l}0.218 \\
0.287\end{array}$ & $\begin{array}{l}\text { O.073 } \\
\text { O-I } 44\end{array}$ & $\begin{array}{l}\text { I Io } \\
\text { I6o }\end{array}$ \\
\hline 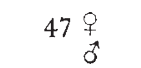 & $\begin{array}{l}0.008 \\
0.018\end{array}$ & $\mid \begin{array}{l}0.000 \\
0.004\end{array}$ & $\begin{array}{l}0.008 \\
0.011\end{array}$ & \begin{tabular}{|l|}
$0 \cdot 083$ \\
$0 \cdot 044$
\end{tabular} & $\begin{array}{l}0.090 \\
0.196\end{array}$ & $\begin{array}{l}0 \cdot 150 \\
0 \cdot 133\end{array}$ & $\begin{array}{l}0.263 \\
0.255\end{array}$ & $\begin{array}{l}0.353 \\
0.302\end{array}$ & $\begin{array}{l}0 \cdot 045 \\
0 \cdot 037\end{array}$ & $\begin{array}{l}133 \\
27 \mathrm{I}\end{array}$ \\
\hline $60 \frac{9}{3}$ & $\begin{array}{l}0.005 \\
0.015\end{array}$ & $\begin{array}{l}0.005 \\
0.000\end{array}$ & $\begin{array}{l}\text { o.005 } \\
\text { o.015 } 5\end{array}$ & $\begin{array}{l}0.036 \\
0.064\end{array}$ & $\begin{array}{l}0 \cdot 164 \\
0 \cdot 223\end{array}$ & 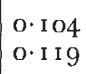 & $\begin{array}{l}0 \cdot 268 \\
0 \cdot 223\end{array}$ & $\begin{array}{l}0.358 \\
0.297\end{array}$ & $\begin{array}{l}0.056 \\
0 \cdot 043\end{array}$ & $\begin{array}{l}444 \\
327\end{array}$ \\
\hline & & & & & & & & & Total & 2986 \\
\hline
\end{tabular}

TABLE $3(b)$

Frequency of colour pattern genotypes in samples from population $B$

\begin{tabular}{|c|c|c|c|c|c|c|c|c|c|c|}
\hline \multirow{2}{*}{$\begin{array}{l}\text { Days after } \\
\text { hatching }\end{array}$} & \multicolumn{9}{|c|}{ Genotype } & \multirow{2}{*}{$\begin{array}{l}\text { No. in } \\
\text { sample }\end{array}$} \\
\hline & $\begin{array}{l}a r \\
a r\end{array}$ & $a n$ & $a t$ & $n n$ & $n r$ & $n t$ & $r r$ & $r t$ & $t t$ & \\
\hline $15 \frac{9}{0}$ & $\begin{array}{l}0.040 \\
0.029\end{array}$ & $\begin{array}{l}0.074 \\
0.044\end{array}$ & $\begin{array}{l}0.029 \\
0.025\end{array}$ & $\begin{array}{l}0.177 \\
0.127\end{array}$ & $\begin{array}{l}0.217 \\
0.284\end{array}$ & $\begin{array}{l}0.17 \mathrm{I} \\
0.127\end{array}$ & $\begin{array}{l}0.097 \\
0.088\end{array}$ & $\begin{array}{l}0.13 \mathrm{I} \\
0.25^{0}\end{array}$ & \begin{tabular}{|l|}
0.063 \\
$0 \cdot 025$
\end{tabular} & $\begin{array}{l}\text { I } 75 \\
204\end{array}$ \\
\hline 22 早 & $\begin{array}{l}0.007 \\
0.016\end{array}$ & $\begin{array}{l}0 \cdot 007 \\
0 \cdot 005\end{array}$ & $\begin{array}{l}0.014 \\
0.005\end{array}$ & $\begin{array}{l}0 \cdot 10 I \\
0 \cdot 08 I\end{array}$ & $\begin{array}{l}0 \cdot 246 \\
0 \cdot 243\end{array}$ & $\begin{array}{l}0.123 \\
0.103\end{array}$ & $\begin{array}{l}0 \cdot 109 \\
0 \cdot 178\end{array}$ & $\begin{array}{l}0.362 \\
0.3^{1} 3\end{array}$ & \begin{tabular}{|l|}
0.029 \\
0.054
\end{tabular} & $\begin{array}{l}138 \\
185\end{array}$ \\
\hline $29 \stackrel{9}{9}$ & $\begin{array}{l}0.011 \\
0.019\end{array}$ & $\begin{array}{l}0.027 \\
0.014\end{array}$ & $\begin{array}{l}0.016 \\
0.019\end{array}$ & $\begin{array}{l}0.080 \\
0.088\end{array}$ & $\begin{array}{l}\text { O. I } 60 \\
0 \cdot 19 I\end{array}$ & $\begin{array}{l}0.218 \\
0.112\end{array}$ & $\begin{array}{l}0.170 \\
0.205\end{array}$ & $\begin{array}{l}0.271 \\
c \cdot 302\end{array}$ & $\begin{array}{l}0.048 \\
0 \cdot 05 \mathrm{I}\end{array}$ & $\begin{array}{l}\text { I } 88 \\
215\end{array}$ \\
\hline 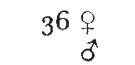 & $\begin{array}{l}0 \cdot 004 \\
0 \cdot 008\end{array}$ & $\begin{array}{l}0 \cdot 000 \\
0 \cdot 000\end{array}$ & $\begin{array}{l}0.013 \\
0.018\end{array}$ & $\begin{array}{l}0 \cdot 100 \\
0 \cdot 083\end{array}$ & $\begin{array}{l}0.266 \\
0.217\end{array}$ & $\begin{array}{l}0.262 \\
0.179\end{array}$ & $\begin{array}{l}\text { O. I } 44 \\
\text { O. I } 89\end{array}$ & $\begin{array}{l}0.153 \\
0.240\end{array}$ & $\begin{array}{l}0 \cdot 057 \\
0.066\end{array}$ & $\begin{array}{l}229 \\
396\end{array}$ \\
\hline $44 \underset{9}{9}$ & $\begin{array}{l}0.014 \\
0 \cdot 005\end{array}$ & $\begin{array}{l}0.014 \\
0.005\end{array}$ & $\begin{array}{l}0.014 \\
0.005\end{array}$ & $\begin{array}{l}0.096 \\
0.067\end{array}$ & $\begin{array}{l}0.228 \\
0.264\end{array}$ & $\begin{array}{l}0.207 \\
0.129\end{array}$ & $\begin{array}{l}0.145 \\
0.218\end{array}$ & $\begin{array}{l}0.207 \\
0.285\end{array}$ & $\begin{array}{l}0 \cdot 076 \\
0 \cdot 021\end{array}$ & $\begin{array}{l}\text { I } 45 \\
\text { I } 93\end{array}$ \\
\hline $\begin{array}{rl}56 & q \\
0 & 0\end{array}$ & $\begin{array}{l}0 \cdot 000 \\
0 \cdot 009\end{array}$ & $\begin{array}{l}0 \cdot 000 \\
0 \cdot 000\end{array}$ & $\begin{array}{l}0.000 \\
0.000\end{array}$ & $\begin{array}{l}0.034 \\
0.073\end{array}$ & $\begin{array}{l}0.180 \\
0.229\end{array}$ & $\begin{array}{l}0.1122 \\
0.110\end{array}$ & $\begin{array}{l}0.416 \\
0.229\end{array}$ & $\begin{array}{l}0.180 \\
0.294\end{array}$ & $\begin{array}{l}0.079 \\
0.055\end{array}$ & $\begin{array}{r}89 \\
109\end{array}$ \\
\hline & & & & & & & & & Total & 2266 \\
\hline
\end{tabular}


The data indicate that in each population:

(a) genotypes with the albomedia allele $\left(F^{a}\right)$ were rare. At hatching, their total proportion did not exceed I5 per cent. of the population and after about 8 weeks it declined to less than 3 per cent.;

(b) the proportion of rubiginosa $\left(F^{r} F^{r}\right)$ increased gradually;

(c) the relative frequency of $F^{n} F^{n}$ decreased whereas $F^{n} F^{r}$ and $F^{n} F^{t}$ either remained constant or decreased in frequency;

(d) the relative frequency of $F^{r} F^{t}$ and $F^{t} F^{t}$ either increased or remained stationary.

Colour pattern gene frequencies estimated from the data in table 3 are given in table $4 . F^{a} F^{a}$ and $F^{a} F^{r}$ are not distinguishable phenotypically and therefore gene frequencies of $F^{a}$ and $F^{r}$ cannot be accurately determined from the data unless relative selective values and the type of mating system are known. However, the albomedia phenotype was fairly rare in all populations, and if mating were random the proportion of homozygotes would be negligible in comparison with the heterozygotes. For example, if $F^{r}=0.5$ and $F^{a}=$ O०OI the proportion of $F^{a} F^{r}: F^{a} F^{a}$ would be Ioo:I, assuming random mating and no differential selection. In calculating the gene frequencies, all individuals with this phenotype were assumed to have the $F^{a} F^{r}$ genotype.

The relative frequency of $F^{a}$ was less than io per cent. in each population and in fact it rarely exceeded 5 per cent. In both populations the frequency of this allele gradually decreased to $\mathrm{I}-2$ per cent. during the first 8 weeks. Although the data indicate that selection was acting against this gene during the active stages of the first generation, there must be counter-selection favouring it either during the egg stage or, perhaps, towards the latter part of the season, otherwise a rather high mutation rate would be required to maintain the gene frequency anywhere near the observed level. The proportion of $F^{r}$ increased at the expense of $F^{n}$ in both populations; $F^{t}$ was relatively constant among females but showed some increase among males during the fourth and fifth instars.

The changes in colour pattern frequency which have been reported are considerable. Are these changes due to natural selection and, if so, are the different instars subject to different selective forces? If the data already presented in table 3 are arranged according to instars, it is found that similar changes in genotypic frequencies occur. This is because the successive samples reflected to a large extent the progression in development of first instars through to adults.

In order to investigate the nature of changes in genotypic frequencies, an analysis was carried out treating the colour pattern frequencies among the instars for each sample separately, i.e. each sample was partitioned with respect to sex, colour pattern and instar. 
In table 5 are given the numbers of $F^{n} F^{r}, F^{n} F^{t}, F^{r} F^{r}$ and $F^{r} F^{t}$ for both sexes observed in those samples where at least two different instars occurred. The analysis of the four colour pattern genotypes for both

TABLE $4(a)$

Frequency of colour pattern genes in the samples from population $A$

\begin{tabular}{|c|c|c|c|c|c|}
\hline $\begin{array}{l}\text { Days after } \\
\text { hatching }\end{array}$ & $F^{a}$ & $F^{n}$ & $F^{r}$ & $F^{t}$ & $2 \mathcal{N}$ \\
\hline $4 \underset{8}{9}$ & $\begin{array}{l}0.056 \\
0.064\end{array}$ & $\begin{array}{l}0.379 \\
0.360\end{array}$ & $\begin{array}{l}0 \cdot 325 \\
0 \cdot 349\end{array}$ & $\begin{array}{l}0 \cdot 240 \\
0 \cdot 226\end{array}$ & $\begin{array}{l}372 \\
358\end{array}$ \\
\hline I I $\begin{array}{r}q \\
\delta\end{array}$ & $\begin{array}{l}0.043 \\
0.064\end{array}$ & $\begin{array}{l}0.407 \\
0.369\end{array}$ & $\begin{array}{l}0.332 \\
0.347\end{array}$ & $\begin{array}{l}0 \cdot 19^{8} \\
0 \cdot 220\end{array}$ & $\begin{array}{l}398 \\
404\end{array}$ \\
\hline 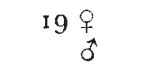 & $\begin{array}{l}0.03^{8} \\
0.049\end{array}$ & $\begin{array}{l}0.317 \\
0.294\end{array}$ & $\begin{array}{l}0.3^{82} \\
0 \cdot 394\end{array}$ & $\begin{array}{l}0 \cdot 263 \\
0 \cdot 263\end{array}$ & $\begin{array}{l}44^{2} \\
494\end{array}$ \\
\hline 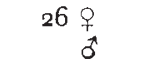 & $\begin{array}{l}0.033 \\
0.05^{2}\end{array}$ & $\begin{array}{l}0.333 \\
0.24^{I}\end{array}$ & $\begin{array}{l}0.374 \\
0.363\end{array}$ & $\begin{array}{l}0.260 \\
0.344\end{array}$ & $\begin{array}{l}270 \\
344\end{array}$ \\
\hline $33 \stackrel{9}{9}$ & $\begin{array}{l}0.036 \\
0.025\end{array}$ & $\begin{array}{l}0 \cdot 300 \\
0 \cdot 253\end{array}$ & $\begin{array}{l}0.350 \\
0.375\end{array}$ & $\begin{array}{l}0 \cdot 314 \\
0 \cdot 347\end{array}$ & $\begin{array}{l}220 \\
320\end{array}$ \\
\hline $47 \underset{0}{9}$ & $\begin{array}{l}0.008 \\
0.017\end{array}$ & $\begin{array}{l}0.203 \\
0 \cdot 210\end{array}$ & $\begin{array}{l}0.489 \\
0.513\end{array}$ & $\begin{array}{l}0 \cdot 300 \\
0 \cdot 260\end{array}$ & $\begin{array}{l}266 \\
542\end{array}$ \\
\hline $\begin{array}{rl}60 & q \\
0 \\
0\end{array}$ & $\begin{array}{l}0.007 \\
0.015\end{array}$ & $\begin{array}{l}0 \cdot 172 \\
0 \cdot 235\end{array}$ & $\begin{array}{l}0.53^{2} \\
0.49^{1}\end{array}$ & $\begin{array}{l}0 \cdot 289 \\
0 \cdot 258\end{array}$ & $\begin{array}{l}888 \\
654\end{array}$ \\
\hline
\end{tabular}

TABLE $4(b)$

Frequency of colour pattern genes in the samples from population $B$

\begin{tabular}{|c|c|c|c|c|c|}
\hline $\begin{array}{l}\text { Days after } \\
\text { hatching }\end{array}$ & $F^{a}$ & $F^{n}$ & $F^{r}$ & $F^{t}$ & $2 \mathcal{N}$ \\
\hline I $5 \begin{array}{r}\text { P } \\
\hat{\delta}\end{array}$ & $\begin{array}{l}0 \cdot 071 \\
0 \cdot 049\end{array}$ & $\begin{array}{l}0 \cdot 409 \\
0 \cdot 355\end{array}$ & $\begin{array}{l}0 \cdot 291 \\
0 \cdot 370\end{array}$ & $\begin{array}{l}0.229 \\
0.226\end{array}$ & $\begin{array}{l}350 \\
408\end{array}$ \\
\hline $22 \underset{0}{9}$ & $\begin{array}{l}0.014 \\
0.013\end{array}$ & $\begin{array}{l}0.290 \\
0.257\end{array}$ & $\begin{array}{l}0.417 \\
0.465\end{array}$ & $\begin{array}{l}0.279 \\
0.265\end{array}$ & $\begin{array}{l}279 \\
370\end{array}$ \\
\hline $\begin{array}{r}29+9 \\
\delta \\
\delta\end{array}$ & $\begin{array}{l}0.027 \\
0.026\end{array}$ & $\begin{array}{l}0 \cdot 282 \\
0 \cdot 246\end{array}$ & $\begin{array}{l}0 \cdot 391 \\
0 \cdot 460\end{array}$ & $\begin{array}{l}0 \cdot 300 \\
0 \cdot 268\end{array}$ & $\begin{array}{l}37^{6} \\
43^{\circ}\end{array}$ \\
\hline $\begin{array}{r}36 \text { 早 } \\
\text { o }\end{array}$ & $\begin{array}{l}0.009 \\
0.012\end{array}$ & $\begin{array}{l}0 \cdot 365 \\
0 \cdot 282\end{array}$ & $\begin{array}{l}0 \cdot 355 \\
0 \cdot 4^{22}\end{array}$ & $\begin{array}{l}0.27 \mathrm{I} \\
0.284\end{array}$ & $\begin{array}{l}45^{8} \\
79^{2}\end{array}$ \\
\hline $44 \frac{9}{\delta}$ & $\begin{array}{l}0.021 \\
0 \cdot 008\end{array}$ & $\begin{array}{l}0 \cdot 321 \\
0 \cdot 267\end{array}$ & $\begin{array}{l}o \cdot 369 \\
0 \cdot 495\end{array}$ & $\begin{array}{l}0.289 \\
0.230\end{array}$ & $\begin{array}{l}290 \\
3^{86}\end{array}$ \\
\hline $56 \stackrel{9}{9}$ & $\begin{array}{l}0.000 \\
0.005\end{array}$ & $\begin{array}{l}0.180 \\
0.243\end{array}$ & $\begin{array}{l}0.596 \\
0 \cdot 495\end{array}$ & $\begin{array}{l}0.224 \\
0.257\end{array}$ & $\begin{array}{l}\text { I } 78 \\
218\end{array}$ \\
\hline
\end{tabular}

populations is summarised in table 6 . The chi-square test of significance used in the analysis was derived by Fisher (see Holt, 1947). The chi-square tests the significance of the regression of colour pattern frequency on instar and takes into account the actual numbers in each sample. 


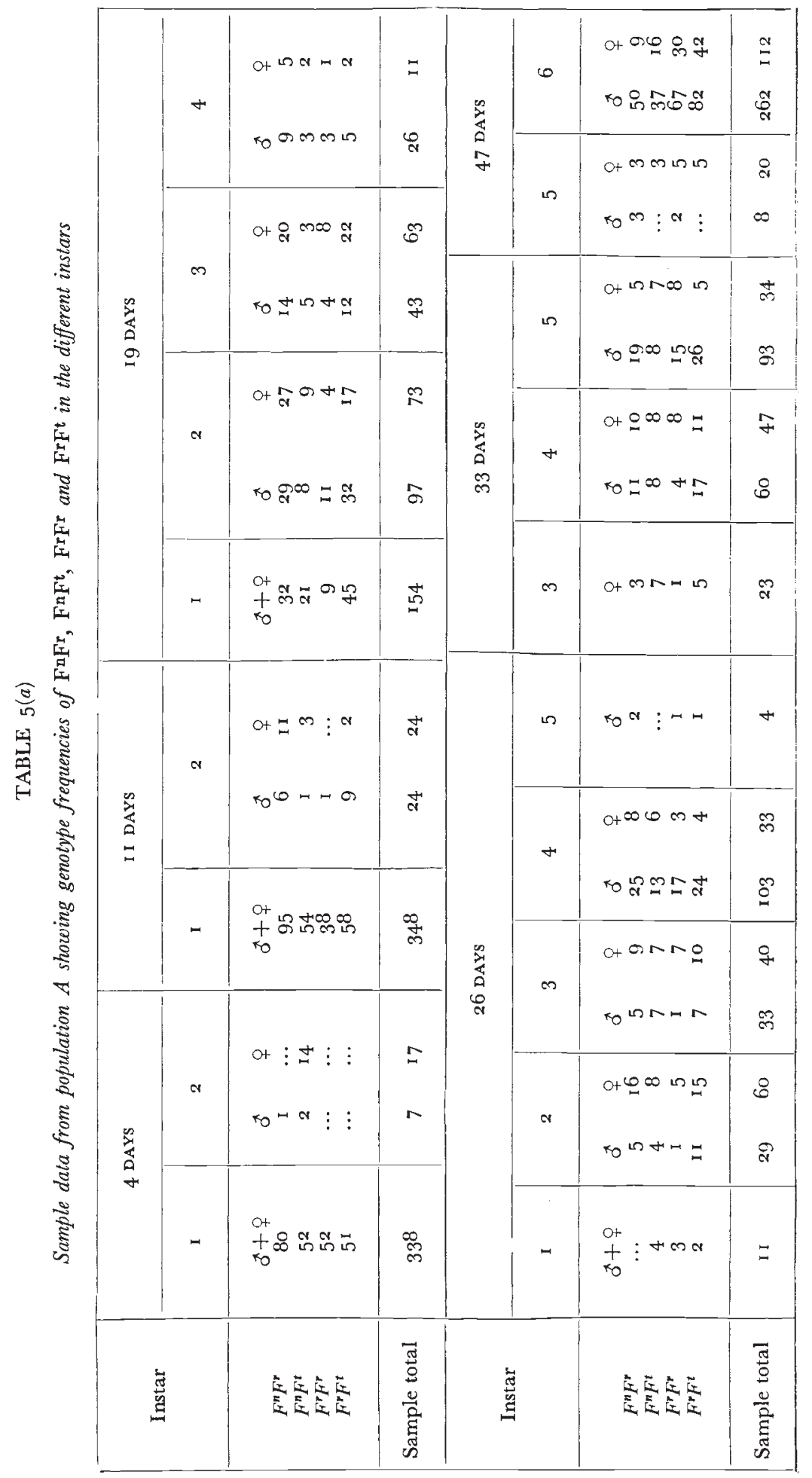


GENE FREQUENGIES IN A LOCUST

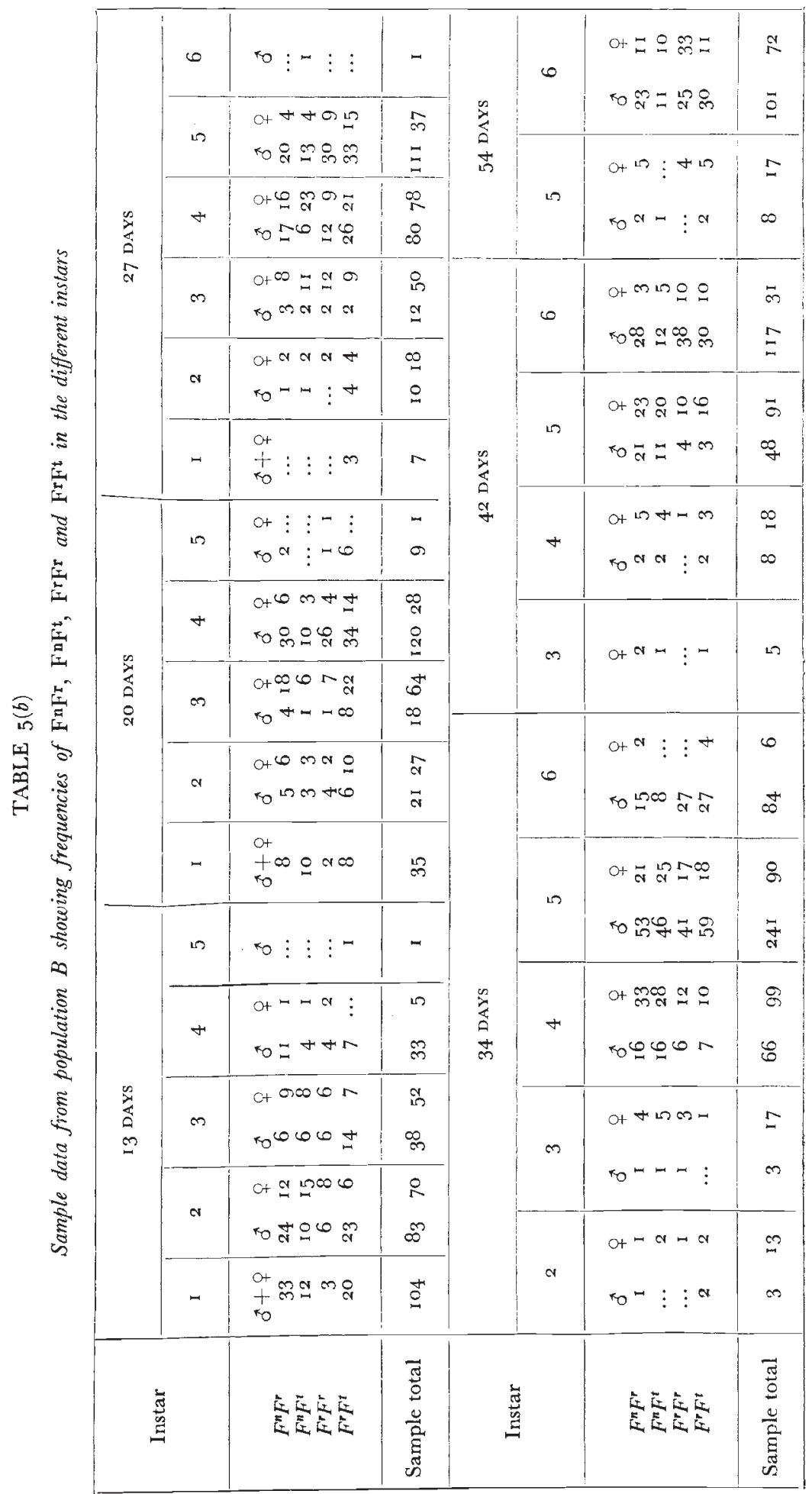


TABLE 6(a)

Statistical analysis of the genotypic frequencies of $\mathrm{F}^{\mathrm{n}} \mathrm{F}^{\mathrm{r}}, \mathrm{F}^{\mathrm{n}} \mathrm{F}^{\mathrm{t}}, \mathrm{Fr}^{\mathrm{r}} \mathrm{Fr}^{\mathrm{r}}$ and $\mathrm{F}^{\mathrm{r}} \mathrm{F}^{\mathrm{t}}$ in population $A$

\begin{tabular}{|c|c|c|c|c|c|c|c|c|}
\hline \multirow{2}{*}{$\begin{array}{l}\text { Sample } \\
\text { (days after } \\
\text { hatching) }\end{array}$} & \multicolumn{4}{|c|}{ MALLES } & \multicolumn{4}{|c|}{ FEMALES } \\
\hline & $\left(q A-p A^{1}\right)$ & $p q \Sigma(a-\bar{a})^{2}$ & $\begin{array}{l}\text { Chi- } \\
\text { square }\end{array}$ & D.F. & $\left(q A-p A^{1}\right)$ & $p q \Sigma(a-\bar{a})^{2}$ & $\begin{array}{l}\text { Chi- } \\
\text { square }\end{array}$ & D.F. \\
\hline \multirow[t]{3}{*}{$\begin{array}{c}\text { Genotype: } F^{n} F^{r} \\
\text { I I } \\
\text { I9 } \\
26 \\
33 \\
47\end{array}$} & $\begin{array}{l}-0.5026 \\
+15.4875 \\
+6.0473 \\
+0.7647 \\
-1.4296\end{array}$ & $\begin{array}{r}4 \cdot 2592 \\
56 \cdot 1672 \\
18 \cdot 5046 \\
5 \cdot 7480 \\
1 \cdot 2250\end{array}$ & $\begin{array}{l}0 \cdot 06 \\
4 \cdot 27 \\
1 \cdot 98 \\
0 \cdot 10 \\
1 \cdot 67\end{array}$ & $\begin{array}{l}\text { I } \\
\text { I } \\
\text { I } \\
\text { I } \\
\text { I }\end{array}$ & $\begin{array}{r}+4 \cdot 1613 \\
+17 \cdot 255^{8} \\
-1 \cdot 3007 \\
+\quad 0.0962 \\
-1.1818\end{array}$ & $\begin{array}{r}4 \cdot 5756 \\
49 \cdot 3308 \\
\mathrm{I} 6 \cdot 3310 \\
7 \cdot 9902 \\
1 \cdot 4017\end{array}$ & $\begin{array}{l}3 \cdot 78 \\
6 \cdot 04 \\
0 \cdot 10 \\
0 \cdot 001 \\
0 \cdot 99\end{array}$ & $\begin{array}{l}\mathrm{I} \\
\mathrm{I} \\
\mathrm{I} \\
\mathrm{I} \\
\mathrm{I}\end{array}$ \\
\hline & $+20 \cdot 3673$ & $85.904^{\circ}$ & $8 \cdot 08$ & 5 & +19.0308 & $79 \cdot 6293$ & 10.91 & 5 \\
\hline & \multicolumn{2}{|c|}{$\begin{array}{l}\text { Total effect within } \\
\text { samples } \\
\text { Heterogeneity between } \\
\text { samples }\end{array}$} & $\begin{array}{l}4 \cdot 83 \\
3 \cdot 25\end{array}$ & $\begin{array}{l}\text { I } \\
4\end{array}$ & \multicolumn{2}{|c|}{$\begin{array}{l}\text { Total effect within } \\
\text { samples } \\
\text { Heterogeneity between } \\
\text { samples }\end{array}$} & $\begin{array}{l}4 \cdot 55 \\
6 \cdot 36\end{array}$ & $\begin{array}{l}\text { I } \\
4\end{array}$ \\
\hline & $\begin{array}{c}+0.9044 \\
-2 \cdot 5484 \\
-3 \cdot 1781 \\
-\quad 7 \cdot 2727 \\
-1 \cdot 7255 \\
\ldots\end{array}$ & $\begin{array}{c}0 \cdot 9240 \\
2 \cdot 7720 \\
29 \cdot 6670 \\
21 \cdot 5418 \\
3 \cdot 3696 \\
\ldots\end{array}$ & $\begin{array}{c}0 \cdot 89 \\
2 \cdot 34 \\
0 \cdot 34 \\
2 \cdot 46 \\
0 \cdot 88 \\
\cdots\end{array}$ & $\begin{array}{l}\text { I } \\
\text { I } \\
\text { I } \\
\text { I } \\
\text { I } \\
\text { W. }\end{array}$ & $\begin{array}{l}+0.3662 \\
+0.9355 \\
-5.9767 \\
-1.4390 \\
-2.2269 \\
-0.1212\end{array}$ & $\begin{array}{r}2 \cdot \text { I I } 87 \\
2 \cdot 9458 \\
9 \cdot 0464 \\
\text { I } 2 \cdot 6280 \\
9 \cdot 3408 \\
2 \cdot 0944\end{array}$ & $\begin{array}{l}0 \cdot 63 \\
0 \cdot 30 \\
3 \cdot 95 \\
0 \cdot 18 \\
0 \cdot 53 \\
0 \cdot 01\end{array}$ & $\begin{array}{l}\text { I } \\
\text { I } \\
\text { I } \\
\text { I } \\
\text { I } \\
\text { I }\end{array}$ \\
\hline & $-13 \cdot 8203$ & $58 \cdot 2744$ & $6 \cdot 91$ & 5 & $-8 \cdot 3409$ & $3^{8 \cdot I} 74^{I}$ & $5 \cdot 60$ & 6 \\
\hline & \multicolumn{2}{|c|}{$\begin{array}{l}\text { Total effect within } \\
\text { samples } \\
\text { Heterogeneity between } \\
\text { samples }\end{array}$} & $\begin{array}{l}3 \cdot 28 \\
3: 63\end{array}$ & $\begin{array}{l}\text { I } \\
4\end{array}$ & \multicolumn{2}{|c|}{$\begin{array}{l}\text { Total effect within } \\
\text { samples } \\
\text { Heterogeneity between } \\
\text { samples }\end{array}$} & $\begin{array}{l}\mathrm{I} \cdot 82 \\
3 \cdot 78\end{array}$ & I \\
\hline \multirow[t]{3}{*}{$\begin{array}{c}\text { Gentoype: } F^{r} \\
\text { I I } \\
\text { I } 9 \\
26 \\
33 \\
47\end{array}$} & $\begin{array}{l}-1 \cdot 5161 \\
+\quad 5 \cdot 9782 \\
+4 \cdot 3333 \\
+3 \cdot 4509 \\
+0 \cdot 0444\end{array}$ & $\begin{array}{r}2 \cdot 1060 \\
22 \cdot 4264 \\
19 \cdot 1780 \\
3 \cdot 9680 \\
1 \cdot 4765\end{array}$ & $\begin{array}{l}I \cdot 09 \\
\mathrm{I} \cdot 59 \\
0 \cdot 98 \\
3 \cdot \text { Oo } \\
0 \cdot 001\end{array}$ & $\begin{array}{l}\text { I } \\
\text { I } \\
\text { I } \\
\text { I } \\
\text { I }\end{array}$ & $\begin{aligned} & \cdots \\
+ & 5.1338 \\
+ & 2 \cdot 6667 \\
+ & 4.4711 \\
+ & 0.2385\end{aligned}$ & $\begin{array}{r}\cdots \\
\text { I } 7 \cdot 0800 \\
\text { I } 4 \cdot 5236 \\
\text { I } 3 \cdot 7700 \\
3 \cdot 4797\end{array}$ & $\begin{array}{l}\ldots \\
\text { I } \cdot 54 \\
0 \cdot 49 \\
\text { I.45 } \\
\text { o. I6 }\end{array}$ & $\begin{array}{l}\text { W } \\
\text { I } \\
\text { I } \\
\text { I } \\
\text { I }\end{array}$ \\
\hline & $+12 \cdot 2907$ & $49 \cdot 1549$ & $6 \cdot 66$ & 5 & $+7 \cdot 1767$ & $4^{8 \cdot 8533}$ & $3 \cdot 64$ & 4 \\
\hline & \multicolumn{2}{|c|}{$\begin{array}{l}\text { Total effect within } \\
\text { samples } \\
\text { Heterogeneity between } \\
\text { samples }\end{array}$} & $\begin{array}{l}3.07 \\
3.59\end{array}$ & $\begin{array}{l}\text { I } \\
4\end{array}$ & \multicolumn{2}{|c|}{$\begin{array}{l}\text { Total effect within } \\
\text { samples } \\
\text { Heterogeneity between } \\
\text { samples }\end{array}$} & $\begin{array}{l}1 \cdot 05 \\
2 \cdot 59\end{array}$ & $\begin{array}{l}\text { I } \\
3\end{array}$ \\
\hline \multirow[t]{3}{*}{$\begin{array}{c}\text { Genotype: } F^{r} F \\
\text { I I } \\
\text { I9 } \\
26 \\
33 \\
47\end{array}$} & $\begin{aligned}+ & 4 \cdot 6774 \\
- & 5 \cdot 6688 \\
- & 4 \cdot 0000 \\
- & 0 \cdot 1373 \\
& \ldots\end{aligned}$ & $\begin{array}{l}3 \cdot 2494 \\
60 \cdot 1750 \\
32 \cdot 2500 \\
7 \cdot 1350 \\
\quad \cdots\end{array}$ & $\begin{array}{l}6 \cdot 73 \\
0 \cdot 53 \\
0 \cdot 50 \\
0 \cdot 003 \\
\ldots\end{array}$ & $\begin{array}{l}\mathrm{I} \\
\mathrm{I} \\
\mathrm{I} \\
\mathrm{I} \\
\mathrm{W}\end{array}$ & $\begin{array}{l}-1 \cdot 8700 \\
+\quad 0 \cdot 7143 \\
-\quad 4 \cdot 4514 \\
-2 \cdot 2211 \\
+\quad 2 \cdot 1212\end{array}$ & $\begin{array}{r}3 \cdot 653 \mathrm{I} \\
50 \cdot 0045 \\
20 \cdot 2680 \\
9 \cdot 0272 \\
3 \cdot 898 \mathrm{I}\end{array}$ & $\begin{array}{l}0 \cdot 96 \\
0 \cdot 001 \\
0 \cdot 98 \\
0 \cdot 55 \\
I \cdot 15\end{array}$ & $\begin{array}{l}\text { I } \\
\text { I } \\
\text { I } \\
\text { I } \\
\text { I }\end{array}$ \\
\hline & $-5 \cdot 1287$ & I O2. 7479 & $7 \cdot 76$ & 4 & -5.7070 & $86 \cdot 8509$ & $3 \cdot 64$ & 5 \\
\hline & \multicolumn{2}{|c|}{$\begin{array}{l}\text { Total effect within } \\
\text { samples } \\
\text { Heterogeneity between } \\
\text { samples }\end{array}$} & $\begin{array}{l}0.26 \\
7 \cdot 50\end{array}$ & $\begin{array}{l}\text { I } \\
3\end{array}$ & \multicolumn{2}{|c|}{$\begin{array}{l}\text { Total effect within } \\
\text { samples } \\
\text { Heterogeneity between } \\
\text { samples }\end{array}$} & $\begin{array}{l}0 \cdot 3^{8} \\
3 \cdot 26\end{array}$ & $\begin{array}{l}1 \\
4\end{array}$ \\
\hline
\end{tabular}


TABLE $6(b)$

Statistical analysis of the genotypic frequencies of $\mathrm{F}^{\mathrm{n}} \mathrm{Fr}, \mathrm{FnF}^{\mathrm{n}}, \mathrm{FrFr}^{\mathrm{r}}$ and $\mathrm{Fr}^{\mathrm{r}}$ in population $B$

\begin{tabular}{|c|c|c|c|c|c|c|c|c|}
\hline \multirow{2}{*}{$\begin{array}{l}\text { Sample } \\
\text { (days after } \\
\text { hatching) }\end{array}$} & \multicolumn{4}{|c|}{ MALES } & \multicolumn{4}{|c|}{ FEMALES } \\
\hline & $\left(q A-p A^{1}\right)$ & $p q \Sigma(a-\bar{a})^{2}$ & $\begin{array}{l}\text { Chi- } \\
\text { square }\end{array}$ & D.F. & $\left(q A-p A^{1}\right)$ & $p q \Sigma(a-\bar{a})^{2}$ & $\begin{array}{l}\text { Chi- } \\
\text { square }\end{array}$ & D.F. \\
\hline \multicolumn{9}{|l|}{ Genotype: $F^{n} F^{r}$} \\
\hline $\begin{array}{l}13 \\
20\end{array}$ & $-5^{\circ} 0000$ & $56 \cdot 0604$ & $0 \cdot 45$ & I & $-12 \cdot 0000$ & $30 \cdot 475^{2}$ & $4 \cdot 70$ & I \\
\hline $\begin{array}{l}20 \\
27\end{array}$ & $\begin{array}{r}+1 \cdot 6552 \\
-0.2066\end{array}$ & $\begin{array}{l}5^{6 \cdot 0286} \\
20 \cdot 8236\end{array}$ & $\begin{array}{l}0.05 \\
0.002\end{array}$ & I & $\begin{array}{l}+1.0260 \\
+0.0327\end{array}$ & $\begin{array}{l}30 \cdot 4876 \\
20 \cdot 0166\end{array}$ & $\begin{array}{l}0.04 \\
0.00\end{array}$ & I \\
\hline 34 & $-6 \cdot 6499$ & $32 \cdot 0733$ & $\mathrm{I} \cdot 3^{8}$ & I & +3.0045 & $33 \cdot 1968$ & 0.27 & I \\
\hline $4^{2}$ & $-6 \cdot 1330$ & II 6424 & $3 \cdot 23$ & I & $-6 \cdot 6828$ & $12 \cdot 1302$ & $3 \cdot 68$ & I \\
\hline \multirow[t]{3}{*}{54} & - $0.165^{2}$ & $\mathrm{I} \cdot 2376$ & 0.02 & I & — I.9439 & $2 \cdot 065^{\circ}$ & $\mathrm{I} \cdot 83$ & $\mathrm{I}$ \\
\hline & - $16 \cdot 4995$ & I $77 \cdot 8659$ & $5 \cdot 13$ & 6 & -16.5635 & І $24 \cdot 6534$ & I0.52 & 6 \\
\hline & \multicolumn{2}{|c|}{$\begin{array}{l}\text { Total effect within } \\
\text { samples } \\
\text { Heterogeneity between } \\
\text { samples }\end{array}$} & $\begin{array}{l}I \cdot 53 \\
3 \cdot 60 \\
\end{array}$ & $\begin{array}{l}\text { I } \\
5 \\
\end{array}$ & \multicolumn{2}{|c|}{$\begin{array}{l}\text { Total effect within } \\
\text { samples } \\
\text { Heterogeneity between } \\
\text { samples }\end{array}$} & $\begin{array}{l}2 \cdot 20 \\
8 \cdot 32 \\
\end{array}$ & $\begin{array}{l}\text { I } \\
5 \\
\end{array}$ \\
\hline \multirow[t]{8}{*}{ Genotyp } & & & & & & & & \\
\hline & - I.5029 & $29 \cdot 75^{64}$ & 0.08 & I & $+4 \cdot 5454$ & $22 \cdot 1088$ & 0.93 & I \\
\hline & $\begin{array}{l}-16.5877 \\
+\quad 2 \cdot 2944\end{array}$ & $\begin{array}{l}30^{\circ} 0268 \\
13 \cdot 0424\end{array}$ & $\begin{array}{l}9.16 \\
0.40\end{array}$ & $\begin{array}{l}\mathrm{I} \\
\mathrm{I}\end{array}$ & $\begin{array}{l}\text {-10.1428 } \\
-0.2896\end{array}$ & $\begin{array}{l}20 \cdot 0736 \\
24 \cdot 9368\end{array}$ & $\begin{array}{l}5.12 \\
0.03\end{array}$ & $\begin{array}{l}\mathrm{I} \\
\mathrm{I}\end{array}$ \\
\hline & $-12 \cdot 1625$ & $3^{2} \cdot 7894$ & $4.5 \mathrm{I}$ & I & $+3 \cdot 1233$ & $29 \cdot 636 \mathrm{I}$ & 0.33 & I \\
\hline & $\begin{array}{r}-5.7515 \\
-0.503\end{array}$ & $6 \cdot 9216$ & $4 \cdot 78$ & I & $-\mathrm{I} \cdot 6207$ & II $\cdot 3229$ & 0.23 & I \\
\hline & & $\mathrm{I} \cdot 0339$ & O.0I & I & . & $\cdots$ & $\cdots$ & $\cdots$ \\
\hline & $-33 \cdot 8295$ & II 3.5705 & 18.94 & 6 & $-4 \cdot 3^{844}$ & $108 \cdot 0782$ & $6 \cdot 64$ & 5 \\
\hline & \multicolumn{2}{|c|}{$\begin{array}{l}\text { Total effect within } \\
\text { samples } \\
\text { Heterogeneity between } \\
\text { samples }\end{array}$} & $\begin{array}{r}10 \cdot 08 \\
8 \cdot 86\end{array}$ & $\begin{array}{l}\text { I } \\
5\end{array}$ & \multicolumn{2}{|c|}{$\begin{array}{l}\text { Total effects within } \\
\text { samples } \\
\text { Heterogeneity between } \\
\text { samples }\end{array}$} & $\begin{array}{l}0 \cdot 18 \\
6 \cdot 46\end{array}$ & I \\
\hline \multirow{7}{*}{$\begin{array}{c}\text { Genotype: } F^{r} I \\
\text { I3 } \\
20 \\
27 \\
34 \\
42 \\
54\end{array}$} & & & & & & & & \\
\hline & $\begin{array}{l}+\mathrm{II} \cdot 0000 \\
+\mathrm{I} 2 \cdot \mathrm{I} 28 \mathrm{I}\end{array}$ & $\begin{array}{l}18 \cdot 6868 \\
42 \cdot 6564\end{array}$ & $\begin{array}{l}6.47 \\
3.45\end{array}$ & $\begin{array}{l}1 \\
\text { I }\end{array}$ & $\begin{array}{r}+10.4545 \\
+\quad 6.9162\end{array}$ & $\begin{array}{l}12.0040 \\
13.8900\end{array}$ & $\begin{array}{l}8.02 \\
3.44\end{array}$ & $\begin{array}{l}\mathrm{I} \\
\mathrm{I}\end{array}$ \\
\hline & $\begin{array}{r}+6.5419 \\
\end{array}$ & $12.735^{\circ}$ & $3 \cdot 36$ & I & $+1 \cdot 5683$ & $2 \mathrm{I} \cdot 0678$ & 0.12 & I \\
\hline & $\begin{array}{l}+16.715^{8} \\
+\quad 8 \cdot 2182\end{array}$ & $24 \cdot 9480$ & I I $\cdot 20$ & I & +4.9179 & 19.0720 & $\begin{array}{l}\mathrm{I} \cdot 27 \\
8 \cdot \mathrm{I}\end{array}$ & I \\
\hline & $\begin{array}{c}+8.2182 \\
\ldots\end{array}$ & $\begin{array}{c}6.453^{8} \\
\cdots\end{array}$ & $10 * 47$ & I & $\begin{array}{r}+7.0500 \\
+\quad 3.0674 \\
\end{array}$ & $\begin{array}{l}0.1200 \\
3.4006\end{array}$ & $\begin{array}{l}0.12 \\
2 \cdot 77\end{array}$ & I \\
\hline & $+54 \cdot 6040$ & I0 5.4794 & $34 \cdot 95$ & 5 & +33.9743 & $76 \cdot 2344$ & $24 \cdot 34$ & 6 \\
\hline & \multicolumn{2}{|c|}{$\begin{array}{l}\text { Total effect within } \\
\text { samples } \\
\text { Heterogeneity between } \\
\text { samples }\end{array}$} & $\begin{array}{r}28 \cdot 27 \\
6 \cdot 68 \\
\end{array}$ & $\begin{array}{l}\text { I } \\
4\end{array}$ & \multicolumn{2}{|c|}{$\begin{array}{l}\text { Total effect within } \\
\text { samples } \\
\text { Heterogeneity between } \\
\text { samples }\end{array}$} & $\begin{array}{r}15.14 \\
9.20\end{array}$ & $\begin{array}{l}\mathrm{I} \\
5\end{array}$ \\
\hline \multirow[t]{3}{*}{$\begin{array}{c}\text { Genotype: } F^{r} F^{t} \\
\text { I3 } \\
20 \\
27 \\
34 \\
42 \\
54\end{array}$} & $\begin{array}{r}+10.2472 \\
+\quad 9.6454 \\
+3.9273 \\
+11.8299 \\
+\quad 5.9480 \\
+\quad 0.3486 \\
\end{array}$ & $\begin{array}{r}13 \cdot 6727 \\
64 \cdot 9026 \\
36 \cdot 5085 \\
29 \cdot 6460 \\
9 \cdot 0384 \\
1 \cdot 4518 \\
\end{array}$ & $\begin{array}{l}7 \cdot 68 \\
\mathrm{I} \cdot 43 \\
0 \cdot 42 \\
4 \cdot 72 \\
3 \cdot 91 \\
0 \cdot 08 \\
\end{array}$ & $\begin{array}{l}\text { I } \\
\text { I } \\
\text { I } \\
\text { I } \\
\text { I } \\
\text { I }\end{array}$ & $\begin{array}{r}5.4070 \\
+12 \cdot 1948 \\
+8 \cdot 1579 \\
+11.8223 \\
+\quad 4.5793 \\
-\quad 1.9439 \\
\end{array}$ & $\begin{array}{r}\text { I } 7 \cdot 9568 \\
37 \cdot 0148 \\
38 \cdot 9648 \\
22 \cdot 0752 \\
\text { 1 1 } 3229 \\
2 \cdot 0636 \\
\end{array}$ & $\begin{array}{l}\mathrm{I} \cdot 63 \\
4 \cdot 02 \\
\mathrm{I} \cdot 7 \mathrm{I} \\
6 \cdot 33 \\
\mathrm{I} \cdot 85 \\
\mathrm{I} \cdot 83 \\
\end{array}$ & $\begin{array}{l}\text { I } \\
\text { I } \\
\text { I } \\
\text { I } \\
\text { I } \\
\text { I }\end{array}$ \\
\hline & $+34^{\circ} 09^{1} 8$ & I $55 \cdot 2199$ & $18 \cdot 24$ & 6 & +29.4034 & I $29 \cdot 398$ I & $17 \cdot 37$ & 6 \\
\hline & \multicolumn{2}{|c|}{$\begin{array}{l}\text { Total effect within } \\
\text { samples } \\
\text { Heterogeneity between } \\
\text { samples }\end{array}$} & $\begin{array}{r}7 \cdot 49 \\
10 \cdot 75 \\
\end{array}$ & $\begin{array}{l}\text { I } \\
5\end{array}$ & \multicolumn{2}{|c|}{$\begin{array}{l}\text { Total effect within } \\
\text { samples } \\
\text { Heterogeneity between } \\
\text { samples }\end{array}$} & $\begin{array}{r}6.68 \\
\text { го.69 } \\
\end{array}$ & $\begin{array}{l}\text { I } \\
5\end{array}$ \\
\hline
\end{tabular}




\section{Interpretation of analysis}

For population A the total maximum likelihood score for $F^{n} F^{r}$ (males) was positive, indicating that within samples the relative frequency of this genotype increased during successive instars. The total effect of this increase within the samples was significant $\left(\chi^{2}=4.83\right.$, I.D.F.) and there was no significant heterogeneity among the samples. Similar changes were recorded for $F^{n} F^{t}$ females. Also for population A, the frequency of $F^{r} F^{\prime}$ increased with the age of individuals but it decreased in the case of $F^{r} F^{l}$ and $F^{n} F^{t}$ respectively. These changes were not significant at the 5 per cent. level.

TABLE 7

Colour pattern genotype frequencies in two samples each from popultions $A$ and $B$ during the second generation

\begin{tabular}{|c|c|c|c|c|c|c|c|c|c|c|}
\hline \multirow{2}{*}{ Sample } & \multicolumn{10}{|c|}{ Colour pattern genotype } \\
\hline & $\begin{array}{c}F^{a} F^{a}, \\
F^{a} F^{r}\end{array}$ & $F^{a} F^{n}$ & $F^{a} F^{t}$ & $F^{n} F^{n}$ & $F^{n} F^{r}$ & $F^{n} F^{t}$ & $F^{r} F^{r}$ & $F^{r} F^{t}$ & $F^{t} F^{t}$ & Total \\
\hline $\begin{array}{r}\text { Population } A \\
23 \text { days } \begin{array}{c}+ \\
0 \\
0 \\
53 \text { days } 0 \\
0 \\
0\end{array}\end{array}$ & $\begin{array}{l}\text { I } \\
4 \\
4 \\
2\end{array}$ & $\begin{array}{l}\text { I } \\
\text { I } \\
\text { O } \\
2\end{array}$ & $\begin{array}{l}0 \\
\text { I } \\
2 \\
6\end{array}$ & $\begin{array}{r}\text { I I } \\
4 \\
\text { I } 7 \\
2 \text { I }\end{array}$ & $\begin{array}{l}31 \\
23 \\
54 \\
53\end{array}$ & $\begin{array}{r}20 \\
6 \\
17 \\
20\end{array}$ & $\begin{array}{l}37 \\
25 \\
98 \\
76\end{array}$ & $\begin{array}{r}32 \\
36 \\
\text { г } 68 \\
\text { I I } 3\end{array}$ & $\begin{array}{r}3 \\
3 \\
24 \\
16\end{array}$ & $\begin{array}{l}\text { I } 36 \\
\text { I03 } \\
384 \\
309\end{array}$ \\
\hline 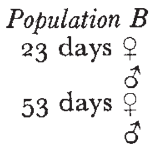 & $\begin{array}{l}\text { I } \\
4 \\
0 \\
\text { I }\end{array}$ & $\begin{array}{l}5 \\
2 \\
I \\
0\end{array}$ & $\begin{array}{l}2 \\
6 \\
0 \\
2\end{array}$ & $\begin{array}{r}88 \\
4.1 \\
6 \\
2\end{array}$ & $\begin{array}{r}\text { I } 38 \\
\text { I } 5 \\
\text { I } 3 \\
\text { I } 4\end{array}$ & $\begin{array}{r}96 \\
72 \\
5 \\
7\end{array}$ & $\begin{array}{r}79 \\
137 \\
22 \\
32\end{array}$ & $\begin{array}{r}108 \\
\text { I } 88 \\
\text { I } 9 \\
3^{\text {I }}\end{array}$ & $\begin{array}{r}9 \\
\text { I } 6 \\
3 \\
4\end{array}$ & $\begin{array}{r}526 \\
58 \mathrm{I} \\
69 \\
93\end{array}$ \\
\hline
\end{tabular}

In population $\mathrm{B}$, the frequencies of both $F^{n} F^{r}$ and $F^{n} F^{t}$ decreased with increasing age of individuals, the change not being significant in the case of $F^{n} F^{t}$ females. On the other hand, $F^{r} F^{r}$ and $F^{r} F^{t}$ showed significant increases.

Natural selection is the most likely explanation for the regression which has been shown to occur for some of the colour pattern genotypes. The homogeneity among samples indicates that there were no real differences in the rate of change in genotype frequency over all samples. One could conclude from this that the relative selective values were fairly constant and were the same for the different stages of development.

Second-generation hatching in these two populations commenced on or just before i8th December i 960 . Samples of nymphs were obtained from both populations on Ioth January I96I-these two samples will be referred to as the 23-day samples. Adults were collected from both populations on 9 th February ig6i and they comprise the 53-day samples. The colour pattern frequencies for the two populations are given in table 7 . 
For population A the colour pattern frequencies among males and females in the 23-day sample were homogeneous. When they are pooled, the total frequencies agree with the expected frequencies generated by the adult gene frequencies in the previous generation assuming random mating $\left(\chi^{2}=7 \cdot 16\right.$ with 5 D.F. $)$.

The proportions of the different instars in this sample were almost equivalent to those in the 26-day sample from the first generation. However, the colour pattern frequencies in the two samples were significantly different both for males and females $\left(\chi^{2}=15.49\right.$ with 5 D.F. for females and $\chi^{2}=19.47$ with 6 D.F. for males). The colour pattern frequencies among adults in first and second generations also differed significantly.

Table 8 gives the colour pattern gene frequencies for the two samples from the second generation, and also the gene frequencies for

TABLE 8

Gene frequencies for the second generation of populations $A$ and $B$. Gene frequencies at the corresponding stages in the first generation are given in brackets

\begin{tabular}{|c|c|c|c|c|}
\hline Sample & $F^{a}$ & $F^{n}$ & $F^{r}$ & $F^{t}$ \\
\hline 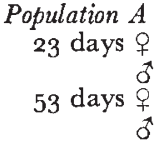 & $\begin{array}{l}0.008(0.033) \\
0.029(0.052) \\
0.008(0.007) \\
0.016(0.015)\end{array}$ & $\begin{array}{ll}0.272 & (0.333) \\
0.185 & (0.241) \\
0.137 & (0.172) \\
0.189 & (0.235)\end{array}$ & $\begin{array}{l}0.5^{07}(0.374) \\
0.548(0.363) \\
0.549(0.532) \\
0.518(0.491)\end{array}$ & $\begin{array}{l}0.213(0.260) \\
0.238(0.344) \\
0.306(0.289) \\
0.277(0.258)\end{array}$ \\
\hline 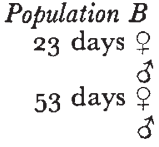 & $\begin{array}{l}0.008(0.027) \\
0.010(0.026) \\
0.007(0.000) \\
0.016(0.005)\end{array}$ & $\begin{array}{l}0.394(0.282) \\
0.233(0.246) \\
0.225(0.180) \\
0.134(0.243)\end{array}$ & $\begin{array}{ll}0.3^{85} & (0.391) \\
0.500 & (0.460) \\
0.551 & (0.596) \\
0.591 & (0.495)\end{array}$ & $\begin{array}{ll}0.213 & (0.300) \\
0.257 & (0.268) \\
0.217 & (0.224) \\
0.259 & (0.257)\end{array}$ \\
\hline
\end{tabular}

the comparable samples from the first generation. The relative frequency of $F^{r}$, which increased from 0.3 to 0.5 during the first generation, did not differ significantly from 0.5 in the two samples from the second generation. The frequency of $F^{n}$ in each sample was less than that in the corresponding samples from the first generation although the difference was not significant. However, as in the first generation, there was a significant decrease in the frequency of this gene between nymphal and adult females. The frequency of $F^{t}$ did not change significantly from its value for adults in the previous generation.

In population $\mathrm{B}$, genotypic frequencies among males and females in the 23-day sample were different and neither agreed with the expected frequencies as derived from the adult gene frequencies for the previous generation. The 29 -day and 56 -day samples from the first generation had an age distribution comparable to the 23-day and 53-day samples respectively from the second generation. The colour pattern frequencies in both generations were homogeneous 202 
except among nymphal females, where the heterogeneity was caused by a rather high frequency of $F^{n} F^{n}$ and $F^{n} F^{r}$ genotypes in the second generation. The gene frequencies in each of the second generation samples agree reasonably well with the gene frequencies in the corresponding first generation samples. However, among females there is a marked discrepancy in the gene frequencies of $F^{n}$ and $F^{r}$ between first generation adults and second generation nymphs. This discrepancy was due largely to the high frequency of the $F^{n} F^{n}$ and $F^{n} F^{r}$ genotypes collected in the 23-day sample.

\section{PROPORTION OF GREEN "FORMS" AMONG THE COLOUR PATTERN GENOTYPES}

The colour patterns already described (Byrne, r967) may include green pigment. The green is located in the integument and may vary from a trace amount usually located on the posterior portion of the disk

TABLE 9

Proportion of green individuals in colour pattern genotype frequencies among females in populations $A$ to $F$

\begin{tabular}{|c|c|c|c|c|c|c|c|c|c|c|}
\hline \multirow{2}{*}{ Population } & \multicolumn{9}{|c|}{ Colour pattern genotype } & \multirow{2}{*}{$\begin{array}{l}\text { Proportion } \\
\text { green in } \\
\text { Population }\end{array}$} \\
\hline & $\begin{array}{l}F^{a} F^{a}, \\
F^{a} F^{r}\end{array}$ & $F^{a} F^{n}$ & $F^{a} F^{t}$ & $F^{n} F^{n}$ & $F^{n} F^{r}$ & $F^{n} F^{t}$ & $F^{r} F^{r}$ & $F^{r} F^{t}$ & $F^{t} F^{t}$ & \\
\hline A & $0 . I_{I}$ & 0.29 & 0.18 & 0.29 & 0.17 & 0.44 & 0.10 & 0.26 & 0.13 & \\
\hline B & 0.08 & 0.20 & 0.14 & 0.27 & 0.08 & 0.35 & 0.02 & 0.24 & 0.09 & 0.18 \\
\hline C & 0.00 & 0.14 & 0.00 & 0.26 & 0.21 & 0.27 & 0.00 & 0.09 & 0.00 & 0.17 \\
\hline D & 0.20 & 0.44 & 0.67 & 0.67 & 0.47 & $0.6 \mathrm{I}$ & 0.39 & $0.5 \mathrm{I}$ & 0.55 & 0.50 \\
\hline E & 0.00 & 0.14 & 0.00 & 0.12 & 0.07 & $0 . I_{I}$ & 0.02 & 0.19 & 0.05 & 0.10 \\
\hline $\bar{F}$ & 0.00 & 1.00 & 0.33 & 0.39 & $0 \cdot 13$ & $0.6 \mathrm{I}$ & 0.12 & 0.51 & 0.00 & 0.31 \\
\hline
\end{tabular}

to an extreme where individuals are completely green except for a few small regions of the body surface. Specimens were classified according to whether or not green pigment was present. The pigment is restricted almost entirely to females, although green males are occasionally found. The proportion of green females among the different colour pattern genotypes for the six populations are given in table 9 .

The percentage of green females in the different populations varied from ro per cent. in $\mathrm{E}$ to almost 50 per cent. in $\mathrm{D} . F^{r} F^{r}$ females consistently had the lowest proportion of green forms. About ro per cent. or less of $F^{r} F^{r}$ individuals were green except in $\mathrm{D}$, where green forms comprised 40 per cent. of $F^{r} F^{r}$ females and 50 per cent. of all females in the population. $F^{n} F^{n}$ and $F^{n} F^{t}$ genotypes had the highest proportion of green phenotypes followed by $F^{r} F^{t}$ and $F^{n} F^{r}$ respectively. The incidence of green forms among the colour pattern genotypes is clearly non-random. A $2 \times 2$ contingency table comparing the incidence of green forms between the $F^{r} F^{r}$ genotype and non $-F^{r} F^{r}$ genotypes is given in table ro. Chi-square for independence equals $8 \mathrm{I} \cdot \mathrm{I}$ with 
I degree of freedom indicating a negative association between the incidence of green forms and the $F^{r} F^{r}$ genotype. When environmental conditions are apparently most favourable for the production of the green pigment, green forms comprise a greater portion of the frequency of $F^{r} F^{r}$ individuals, as in population $\mathrm{D}$.

TABLE Io

$2 \times 2$ contingency table showing a negative association between the incidence of the $\mathrm{Fr} F r$ genotype and the green form among females

\begin{tabular}{|l|c|c|c|}
\hline & $F^{r} F^{r}$ & non- $F^{r} F^{r}$ & Total \\
\hline Green & 68 & 853 & $92 \mathrm{I}$ \\
Non-green & 625 & $24^{6} 3$ & 3088 \\
\hline Total & 693 & $33^{16}$ & 4009 \\
\hline
\end{tabular}

$\chi^{2}=8 \mathrm{I} \cdot \mathrm{I}$ with I D.F.

\section{EFFECT OF DISPERSION AND MIGRATION ON COLOUR PATTERN FREQUENCIES}

During its life-cycle, $C$. terminifera is often exposed to ecologically diverse environments as afforded by oviposition and food-shelter habitats. Some of the nymphs may disperse from the oviposition site and complete their development in the food-shelter habitat. By studying colour pattern frequencies simultaneously in both environments it may be possible to determine whether or not dispersion of nymphs is random with respect to colour pattern. Kettlewell (I958) showed that in Biston betularia there is a marked preference by melanic forms for dark backgrounds when landing, whereas the non-melanics prefer lightcoloured backgrounds. Such a study may also indicate if the relative selective values were the same in both environments.

The area occupied by population B consisted of an oviposition habitat with sandy soil and a food-shelter habitat with black, selfmulching soil. Nymphs started dispersing from the oviposition site to the food-shelter during the third and fourth instar. Samples were collected from each habitat 27 and 34 days after hatching. The colour pattern frequencies for these samples are given in table II. The colour pattern frequencies in the two samples collected on the $27^{\text {th }}$ day were homogeneous $\left(\chi^{2}=3 \cdot 57\right.$ with 5 D.F. for females and $\chi^{2}=7 \cdot 7$ with 5 D.F. for males). It would therefore appear that nymphs disperse at random with respect to colour pattern. However, from the samples collected on the $34^{\text {th }}$ day, there was a significant difference between the two environments in colour pattern frequencies $\left(\chi^{2}=15 \cdot 39\right.$ for females, $\chi^{2}=$ I I 5 for males, each with 5 D.F.). When the samples were obtained on the 27 th day, there were relatively few nymphs in 
O. R. BYRNE

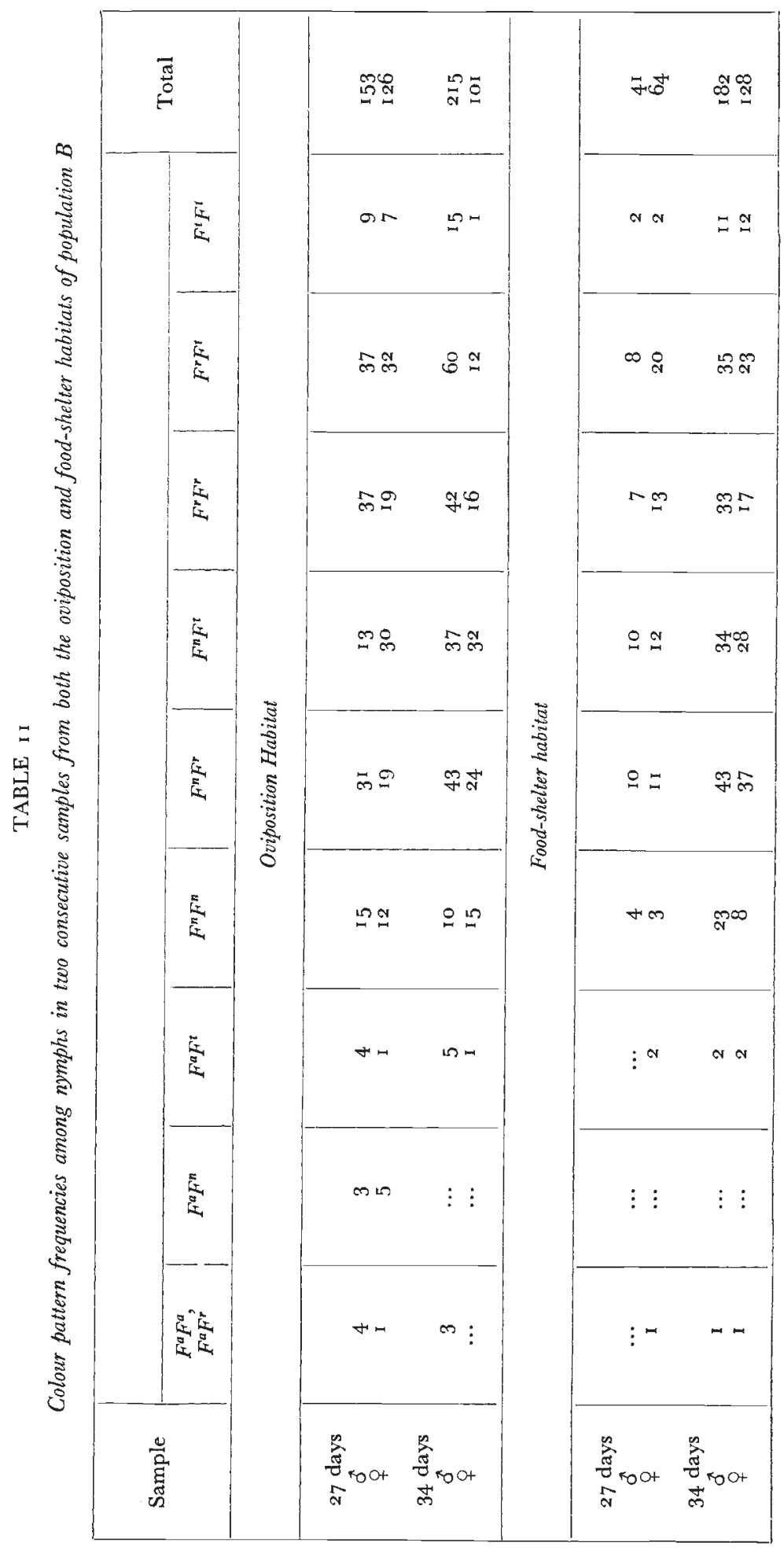


the food-shelter habitat but there was a dense band of them moving across the transitional zone. By the $34^{\text {th }}$ day, the nymphs had become distributed over a large area of the food-shelter habitat. It seems likely that the difference in colour pattern frequencies between the two samples collected on this occasion was the result of different selective forces operating in the two environments. In fact, in the food-shelter habitat, conditions for survival must have been extremely adverse. By the $45^{\text {th }}$ day very few grasshoppers had survived and it was not possible to obtain enough individuals for a reliable sample.

In population $\mathbf{F}$, a large portion of the adults migrated from the habitat as a "loose swarm". Samples which were obtained on 2nd and $14^{\text {th }}$ November 1960 were taken both from migrating and nonmigrating locusts. For each occasion the two samples had similar colour pattern frequencies. (For $2 /$ I I $/ 60, \chi^{2}=6.5$ with 5 D.F. and for $14 /$ I I $/ 60 \chi^{2}=6 \cdot 28$ with 6 D.F.). This suggests that there is no differential migration with respect to colour pattern.

\section{DISCUSSION}

For most of the populations studied, colour pattern frequencies differed between males and females. This discrepancy could result if males and females had different genotypic selective values. If this were so, then greater differences in colour pattern frequencies between the sexes would be expected among adults than among nymphs. This was not observed. Of course, it is possible that opposing selective forces may have been operating at different stages of the life-cycle and this could lead to a similar situation to that reported here.

For nymphs, the relative genotypic frequencies of $F^{n} F^{n}$ and $F^{n} F^{t}$ were generally greater in females than in males from the same sample. The incidence of green forms is higher among females of either of these genotypes than of any other colour pattern genotype. If the presence of green pigment in females improves their adaptation, which may be the case particularly on a green background, then the genotypic selective value would be expected to be higher for females than for males. Presumably, the green pigment would have to be somewhat deleterious in adult females to account for the difference in frequency of these genotypes among fifth instar nymphs and adults. It is likely that green adults are less active as fliers as reported by Rubtzov in Chorthippus albomarginatus and hence more prone to predation, or perhaps green forms are more susceptible to disease and parasitism both of which appear to be more prevalent among adults than nymphs -at least from laboratory studies (Byrne, r967).

Differences in the relative frequencies of colour patterns between the sexes were greater within instars than within samples. For population $\mathrm{A}$, although the frequencies in the samples were homogeneous, they were not homogeneous within each instar. Either males and females do have different selective values or, if they are the same, the 
difference may be due to the fact that females develop at a slower rate than males.

The changes in relative frequency of the different colour patterns during the first generation were rather similar in each of the populations. Colour patterns which were either heterozygous or homozygous for the $F^{a}$ allele were selected against. However, this gene must be favoured by selection at some stage otherwise its frequency could not be maintained except by a very high mutation rate. The $F^{a}$ allele may have survival value either during the later generations or in the egg stage. It could also be favoured by gametic selection.

The increase in frequency of the homozygote $F^{r} F^{r}$ indicates that this colour pattern has a selective advantage during the first generation. To explain this increase in frequency one must consider not only the environment for this particular generation but also that in which the previous generation was reared. These two generations were separated in time by at least five months during which the eggs over-wintered. The ecological characteristics of the habitat at these two different times of the year would be different, particularly with regard to temperature, humidity and vegetation. It is also possible that the ancestors of some of the populations were immigrants from geographically different habitats. The gene frequency of $F^{r}$ among the adult ancestors must have been considerably lower than among adults of the first generation. After hatching of the first generation commenced in October I960, there was a period of Io weeks during which no rainfall occurred. In this period soil moisture was gradually depleted and daily temperatures increased. The pasture plants which were present at the time of hatching were mainly annual species and these had completed their growth. The subsequent death of these plants resulted in a shortage of green food and a change in the background colour of the habitats. Also, the area of bare patches of soil increased considerably. In other words, during the first generation each habitat became increasingly drier and its background colour became relatively more uniform. The rubiginosa $\left(F^{r} F^{r}\right)$ colour pattern is apparently better adapted than other colour patterns to this kind of environment. It is uniformly brown with black mottling and it certainly appears to be less conspicuous than other colour patterns when on backgrounds such as dead plants and exposed areas of soil. The $F^{r} F^{r}$ genotype would most likely have a selective advantage in such an environment against the action of predators. It is also likely that individuals with this colour pattern are physiologically better adapted to withstand dry conditions than are most other genotypes. They may utilise the available moisture more efficiently or perhaps they are better equipped to survive in the absence of an adequate supply of green food. Rubtzov (1935) recorded that the rubiginosa colour pattern in Chorthippus albomarginatus was relatively more abundant in dry than in humid habitats. Descriptions of this colour pattern suggest that its appearance is similar to the $F^{r} F^{r}$ pattern in $C$. terminifera; however, it may well have a different genetical basis. 
During the second generation rainfall was adequate and summer growing plants were abundant in most of the habitats. In this generation the frequency of $F^{r} F^{r}$ in population $\mathrm{A}$ did not change appreciably from its value for adults in the first generation.

No data were obtained on the effect of predation on colour pattern frequencies. However, the capture of specimens by other invertebrate animals was observed on several occasions. These predators were mainly spiders, Psammocharid wasps and Asilid flies. The work of Carrick (1959) on the food and feeding habitats of two species of Ibis suggest that these birds may be important predators of $C$. terminifera. Although visual selection by predators may play a part in maintaining the polymorphism, it is likely that physiological selection is more important.

From inadequate data it would appear that migration does not affect the genetic structure of populations, at least with respect to colour patterns. Migration is an important feature of behaviour in gregarious phases of this locust, and for those populations which colonise habitats similar to Key's outbreak centres its occurrence must be common. Little is known either of the factors which trigger off migration in C. terminifera or of the fate of the migrating swarm. Individuals which leave a population certainly become mixed with those from other populations and they may traverse several hundred miles. The migratory phase is generally short-lived and perhaps the majority of adults die without reproducing. It is unlikely that migrants found new populations which can be maintained for a long period of time. However, it is reasonable to expect new populations to be formed in fairly unfavourable habitats and they may persist for a small number of generations if climatic conditions permit. From the evolutionary viewpoint migration is perhaps an adaptive mechanism operating for the survival of those individuals which remain in their habitat. It is a way of eliminating a high proportion of the numbers in a population when competition for space and food become important for survival. It can be regarded as an environmentally determined density-regulating mechanism acting to regulate population density before all resources are depleted and doing so quite independently of the genetic structure of the population. If this large-scale migration resulted in important changes in the population gene pool, it would impose an unnecessary evolutionary load on the population. Several generations of selection would then be required to equilibrate the gene frequencies to levels approaching those determined by the habitat for the population in a non-migratory phase.

It cannot be inferred from the data whether colour pattern polymorphism is transient or balanced. However, it seems unlikely that multiple alleles would be in a transient polymorphism unless the polymorphism was previously balanced. Marked changes in the selective values could upset the equilibrium and the polymorphism could become transient for one or more of the alleles. Data from a 
number of successive generations are necessary to decide this point. For multiple alleles in a selectively balanced polymorphism, the necessary conditions for a stable equilibrium require that no heterozygote be less fit than both the associated homozygotes (Owen, I953; Kimura, I956; Mandel, I959). It is therefore possible to have a balanced polymorphism without all heterozygotes showing overdominance. The equilibrium conditions assume random mating and constant selective values. Further studies in progress should indicate if the average values for the different genotypes satisfy the conditions for a stable equilibrium.

The studies up to date have revealed a number of problems which require further investigation before the evolutionary significance of colour patterns in this species becomes clear. Field studies throughout a number of different seasons should indicate whether or not those colour patterns which were favoured by selection during the first generation maintained their advantage for the remainder of the season. The importance of predators, particularly the ibis, in maintaining the polymorphism is also being investigated. A study of the effect of different environments on genotypic frequencies in separate populations reared in laboratory cages may indicate if some colour patterns are better adapted physiologically than others, and if so, the basis for the adaption.

\section{SUMMARY}

I. The Australian plague locust, Chortoicetes terminifera, occurs in a wide range of habitats throughout Australia. It is strongly migratory and subject to phase variation.

2. Under field conditions, the generation time is about I I weeks, and three generations may be completed in a year if environmental conditions are favourable.

3. Data on colour pattern frequencies in natural populations were obtained for part of the I96o-6I season. There is evidence that the polymorphism is maintained by selection and that selective values vary from population to population.

4. The effect of natural selection on colour pattern gene frequencies can be studied during all stages of the life-cycle except the egg stage. Data from the first generation in the I960-6 I season suggest that the genotypic selective values were the same for the different instars.

5. At different times in a season, and in different seasons, selective values are likely to be different.

6. Migration appears to be independent of colour pattern genotype.

7. The polymorphism may be maintained primarily through physiological selection, although visual selection by predators could also be important.

Acknowledgments. - The guidance of Professor J. H. Bennett throughout these studies is gratefully acknowledged. Acknowledgment is also due to Professor D. G. 
Catcheside for many helpful comments concerning the manuscript, to Messrs B. Cameron and J. Walker, Trangie, for assistance in field collections, and also to Division of Entomology, C.S.I.R.O., for finance to carry out the field studies. This work was carried out under tenure of a C.S.I.R.O. Senior Studentship within the Genetics Department, University of Adelaide.

\section{REFERENCES}

byrne, o. R. 1966. Polymorphism in the Australian Acridide: 1. Inheritance of colour patterns in the plague locust, Chortoicetes terminifera. Heredity, 22, 561-568.

CARRICK, R. 1959. The food and feeding habits of the straw-necked ibis, Threskiornis spinicollis, and the white ibis, T. molluca, in Australia. C.S.I.R.O. Wild Life Research, 4, 69-92.

FISHER, R. A. 1930. The evolution of dominance in certain polymorphic species. Amer. Nat., 64, 285-406.

Fisher, R. A. 1939. Selective forces in wild populations of Paratettix texanus. Ann. Eugen., 9, 109-123.

HOLT, s, в. 1948. The effect of maternal age on the manifestation of a polydactyl gene in mice. Ann. Eugen., 14, 144-157.

KETTLEWELL, н. в. D. г 956 . A resumé of investigations on the evolution of melanism in the Lepidoptera. Proc. Roy. Soc. London ser. B, 145, 297-303.

KETTLEWELL, H. B. D. 1958. The importance of microenvironment to evolutionary trends in Lepidoptera. The Entomologist, 9I, $214-224$.

kimura, M. 1956. Rules for testing stability of a selective polymorphism. Proc. U.S. Nat. Acad. Sci., 42, 336-340.

MandeL, s. P. H. 1959. The stability of a multiple allelic system. Heredity, 13, 289-302.

owen, A. R. G. 1953. Balanced polymorphism of a multiple allelic series. Proc.

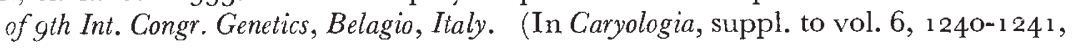
1954.)

RUBtzov, I. A. 1935. Phase variation in non-swarming grasshoppers. Bull. Ent. Res., 26, 499-524. 